\title{
High Energy Raman Solitons in Multimode GRIN Fibers
}

\author{
M. Zitelli ${ }^{1}$, F. Mangini ${ }^{2}$, D.S. Kharenko ${ }^{3}$, A. Niang $^{2}$, S. Wabnitz ${ }^{1,3}$ \\ ${ }^{1}$ Department of Information Engineering, Electronics and Telecommunications (DIET), \\ Sapienza University of Rome, Via Eudossiana 18, 00184 Rome, Italy. \\ ${ }^{2}$ Department of Information Engineering (DII), University of Brescia, Via Branze 38, 25123 Brescia, Italy. \\ ${ }^{3}$ Novosibirsk State University, Pirogova 1, Novosibirsk 630090, Russia. \\ mario.zitelli@uniromal.it
}

\begin{abstract}
We experimentally demonstrate the fission of femtosecond pulses in a GRIN standard fiber, leading to high-energy multimode solitons that undergo huge Raman frequency shifts, and exhibit complex multisoliton dynamics. $\odot 2020$ The Author(s)
\end{abstract}

Nonlinear pulse propagation in multimode (MM) optical fibers is a subject of renewed research interest: MM fibers hold promise to increase the capacity of next-generation communications systems, and to scale up the energy of supercontinuum and fiber laser sources [1-4]. Yet, complex pulse propagation effects in MM fibers remain far from being completely understood. In this work, we consider the high-energy regime of femtosecond pulse propagation in a standard graded-index (GRIN) MM fiber with anomalous dispersion. Intrapulse Raman scattering leads to the fission of the initial pulse into MM solitons, which undergo Raman self-frequency shift (SSFS). The impact of self-imaging on SSFS in GRIN MM fibers has been recently investigated by a simple singlemode model, involving a spatially varying effective mode area [5]. Here we reveal a new pulse propagation regime, where fission of the initial pulse undergoes a complex behavior, leading to decay into several high-energy MM solitons.

The experimental setup to study the generation of high-energy MM solitons consists of an ultra-short laser system, involving a hybrid optical parametric amplifier (OPA) of white-light continuum, pumped by a femtosecond Yb-based laser, generating $120 \mathrm{fs}$ pulses at $1550 \mathrm{~nm}$, with $25 / 100 \mathrm{kHz}$ repetition rate. The laser beam is attenuated by variable ND filters, and focused by a 30,50 or $75 \mathrm{~mm}$ lens, to vary the input beam diameter $\left(1 / e^{2}\right)$ to 18 , 30 and $45 \mu \mathrm{m}$, into a $30 \mathrm{~cm}$ long multimode standard 50/125 GRIN fiber with relative index difference $\Delta=$ 0.0102 and chromatic dispersion $\beta_{2}=-22 \mathrm{ps}^{2} / \mathrm{km}$ at $1550 \mathrm{~nm}$. A micro-lens focuses the output near field in an optical spectrum analyzer (OSA) and a spectrometer, with wavelength range 600-1700 nm and 1100-3000 nm, respectively.

Output spectra have been recorded at different input peak powers (from a few Watts up to several MWs). Fig. 1.a shows the recorded normalized spectra for input peak powers ranging from the linear regime up to 3 MW, with an input beam diameter of $30 \mu \mathrm{m}$. The fission of the input pulse leads to several MM solitons with energies up to $50 \mathrm{~nJ}$, that undergo Raman wavelength shifts above $2 \mu \mathrm{m}$. As can be seen in Fig. 1.a, the Raman shift tends to saturate at high input powers, owing to nonlinear losses originating from fluorescence and higher harmonic scattering over the first few centimeters of the MMF [4].

Individual lobes composing the measured output spectrum can be easily fitted with a $\operatorname{sech}^{2}$ shape, indicating soliton formation. One may thus extract the corresponding soliton energy, peak power, bandwidth, pulsewidth and central wavelength. Experimental spectra could be well reproduced by numerical simulations, using an exact 3D+1- vectorial model including second, third and fourth order dispersion, Kerr and Raman nonlinearities (with fraction $f_{R}=0.18$ ), wavelength-dependent losses of silica fibers, and both polarizations. Fig. 1.b compares the measured wavelength shift of different individual Raman-shifted MM solitons at the fiber output, for a $30 \mu \mathrm{m}$ input beam diameter, as a function of soliton energy. Fig. 1.b shows that successive MM Raman solitons saturate their Raman shift at a different wavelength. In Fig. 2.a the pulsewidth of the two most Raman shifted MM solitons is reported, as a function of their energies. As can be seen, different solitons generated by fission of the input multisoliton pulse tend to maintain equal temporal durations for energies greater than $10 \mathrm{~nJ}$. The soliton pulsewidth remains close to that generated during the fission process.

In Fig. 2.b, the wavelength of the most Raman shifted MM soliton is reported, as a function of the total energy of the input pulse, for different input beam diameters. Fig. 2.b shows that the largest input beam diameter (i.e., the highest input multimode excitation) leads to the smallest SSFS value.

Finally, Fig. 2.c shows the overall order of the set of output MM solitons, calculated as $N^{2} \approx\left(T_{0} \sum_{k} N_{k}^{2} / T_{k}\right)$, where $k=1,2, \ldots, M$ and $M$ is the number of output Raman pulses, $T_{0}=T_{F W H M} / 1.763$ is the input pulse temporal width, and $T_{k}$ and $N_{k}$ are the time widths and the order of the individual output MM solitons, respectively. The 
order of the individual solitons can be calculated from the standard soliton theory, as $N=\sqrt{\gamma P_{0} \tau_{0}^{2} /\left(\left|\beta_{2}\right|\right)}$ [4], where $\beta_{2}$ the fiber dispersion at the respective soliton wavelength, $\gamma$ is the nonlinear coefficient, and $P_{0}$ is the soliton peak power. As can be seen, the overall order of the set of output MM solitons is the highest for smaller input beam diameters, reaching values of up to $N=3$ in the experiment. Specifically, we find that the input laser pulse of sech shape and of soliton order $N$, undergoes fission into several high-order MM Raman solitons with order $N_{k}$ approaching unity; solitons generated by the fission tend to conserve equal pulsewidth; the maximum observed number of output pulses is $M=5$.
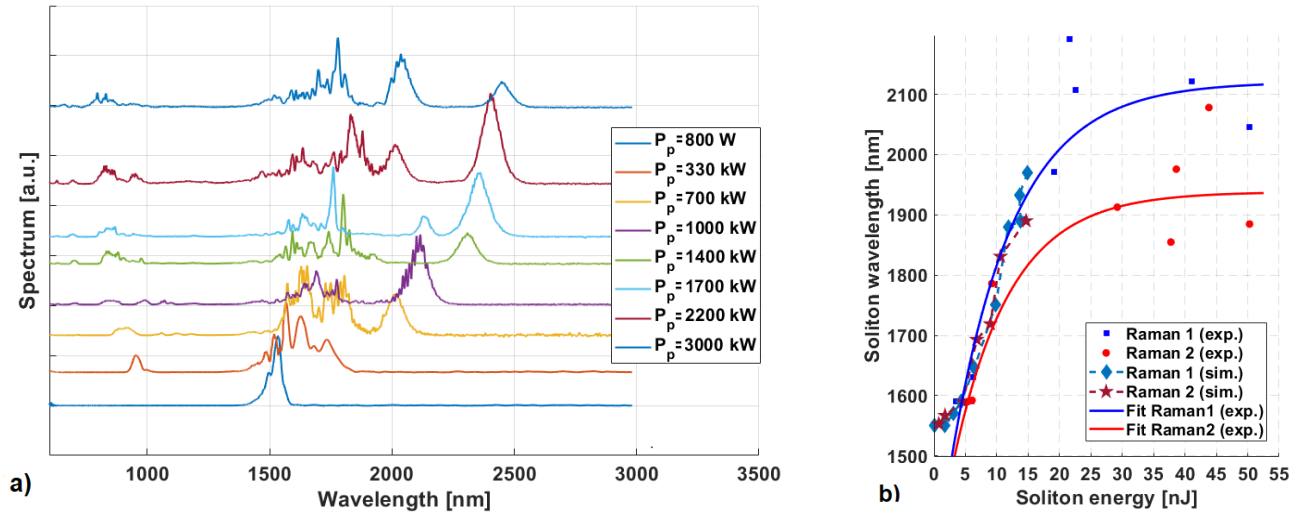

Fig. 1. a) Output spectra at different input powers for $30 \mu \mathrm{m}$ beam diameter. b) Wavelength shift of individual Raman MM solitons vs. soliton energy from numerics and experiments.
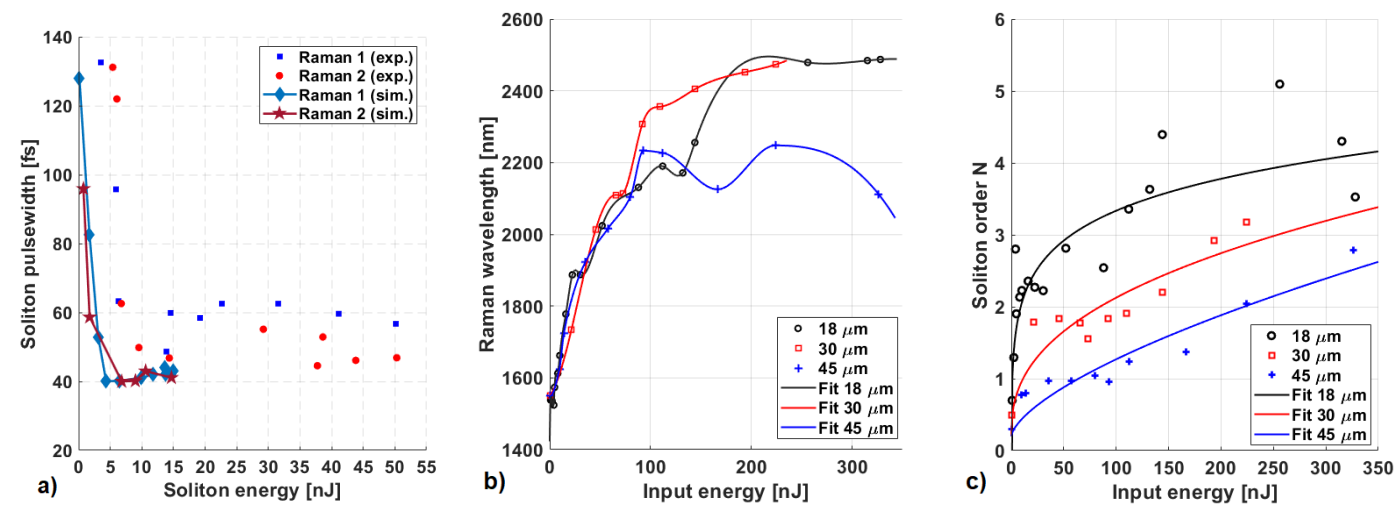

Fig. 2. a) Soliton pulsewidth vs. soliton energy. b) Wavelength of MM Raman solitons, for 18, 30, $45 \mu \mathrm{m}$ input beam diameters, vs. input energy. c) Overall soliton order (for 18, 30, $45 \mu \mathrm{m}$ input beam diameters) vs. input energy.

In summary, we investigated a new regime of high-energy MM soliton generation by Raman-induced fission, which leads to stable emission of a soliton wavelength multiplex with similar pulsewidth. These results are of importance for the development of novel soliton laser sources in the near and mid-infrared.

This work was supported by the European Research Council (grant No. 740355), and by the Russian Ministry of Science and Education, (grant No. 14.Y26.31.0017).

\section{References}

1. W.H. Renninger et al., "Optical solitons in graded-index multimode fibres," Nat. Commun. 4, 1719 (2013).

2. L.G. Wright, et al., "Spatiotemporal dynamics of multimode optical solitons," Opt. Express 23, 3492 (2015).

3. L.G. Wright, et al., "Controllable spatiotemporal nonlinear effects in multimode fibres," Nature Photonics 9, 306 (2015).

4. K. Krupa, et al., "Multimode nonlinear fiber optics, a spatiotemporal avenue," APL Photonics 11, 110901 (2019).

5. A.S. Ashan and G.P. Agrawal, "Spatio-temporal enhancement of Raman-induced frequency shift in gradedindex multimode fibers,” Opt. Lett. 44, 2637 (2019). 